\title{
Mixed Features in Depression
}

\section{The Unmet Needs of Diagnostic and Statistical Manual of Mental Disorders Fifth Edition}

\author{
Isabella Pacchiarotti, $M D, \mathrm{PhD}^{\mathrm{a}}$, Giorgio D. Kotzalidis, $\mathrm{MD}, \mathrm{PhD}^{\mathrm{b}}$, \\ Andrea Murru, MD, PhD ${ }^{a}$, Lorenzo Mazzarini, MD, PhD ${ }^{\mathrm{b}}$, \\ Chiara Rapinesi, MD ${ }^{\mathrm{b}}$, Marc Valentí, MD, $\mathrm{PhD}^{\mathrm{a}}$, \\ Gerard Anmella, MDa ${ }^{a}$, Susana Gomes-da-Costa, MDa, \\ Anna Gimenez, $\mathrm{MD}^{\mathrm{a}}$, Cristian Llach, $\mathrm{MD}^{\mathrm{a}}$, Giulio Perugi, MD, $\mathrm{PhD}^{\mathrm{C}}$, \\ Eduard Vieta, MD, PhD ${ }^{a, *}$, Norma Verdolini, $M D, \mathrm{PhD}^{\mathrm{a}}$
}

\section{KEYWORDS}

- Major depressive disorder • Bipolar disorder • DSM-5 • Mixed features specifier

- Nonoverlapping symptoms • Overlapping symptoms

\section{KEY POINTS}

- The identification of mixed features during a major depressive episode is important due to the worse course and treatment issues associated with this condition.

- Diagnostic and Statistical Manual of Mental Disorders Fifth Edition mixed features specifier criteria are controversial, because it includes typical manic symptoms, whereas it excludes overlapping excitatory symptoms that are frequently reported in mixed depression.

- Psychomotor agitation, mood lability, and aggressiveness are the new proposed criteria based on the results of the Bipolar Disorders: Improving Diagnosis, Guidance and Education-II-Mix study.

- Several clinical and course indicators of bipolarity were found to be associated with the presence of mixed characteristics, mainly overlapping excitatory symptoms, during a major depressive episode.

\footnotetext{
a Barcelona Bipolar and Depressive Disorders Program, Hospital Clinic, University of Barcelona, Institute of Neuroscience, IDIBAPS, CIBERSAM, 170 Villarroel st, 12-0, Barcelona, Catalonia 08036, Spain; ${ }^{b}$ Neurosciences, Mental Health, and Sensory Organs (NESMOS) Department, Faculty of Medicine and Psychology, Sapienza University, Sant'Andrea University Hospital, UOC Psichiatria, Via di Grottarossa 1035-1039, Rome 00189, Italy; ' Department of Clinical and Experimental Medicine, University of Pisa, Via Roma 67, Pisa 56100, Italy

* Corresponding author. Bipolar Disorders Unit, Institute of Neuroscience, IDIBAPS, CIBERSAM, Hospital Clínic de Barcelona, c/Villarroel, 170, 12-0, Barcelona 08036, Spain.

E-mail address: EVIETA@clinic.cat
} 


\section{INTRODUCTION}

The identification of mixed features in bipolar disorder (BD) and major depressive disorder (MDD) is an open challenge in psychiatry, because an accurate diagnosis is a prerequisite for the initiation of adequate therapeutic approaches. ${ }^{1-4}$ Although the importance of mixed states was increasingly recognized in the last decades, the Kraepelinian concept of a continuum between manic and depressive states was not incorporated into major psychiatric diagnostic systems such as the Diagnostic and Statistical Manual of Mental Disorders Third Edition (DSM-III), DSM-III-R, DSM-IV, DSM-IV-TR, ${ }^{5-8}$ and the International Classification of Diseases, Tenth Edition. ${ }^{9}$ Substantial changes to the diagnosis of BD mixed state were made in the DSM-5.

The DSM-IV-defined categorical approach of diagnosing mixed states (ie, a period of 1 week when the criteria are met for both a manic episode and a major depressive episode [MDE] nearly every day) has been replaced in DSM-5 by the dimensional mixed features specifier (MFS). ${ }^{10}$ Despite the introduction of the MFS, substantial controversy still remains as to whether the definition proposed reflects the empirical evidence. ${ }^{11,12}$

\section{MAJOR DEPRESSIVE EPISODE WITH MIXED FEATURES}

The importance of promptly recognizing the presence of mixed characteristics during an MDE is mostly due to clinical variables associated with greater severity, namely the higher number of episodes ${ }^{13}$; the lower response or switch with antidepressants $(\mathrm{ADs})^{14}$; the high prevalence of psychiatric comorbidities such as anxiety, substance use, ${ }^{13}$ and borderline personality disorder ${ }^{15}$; the increased risk of suicide ${ }^{16}$; worse psychosocial functioning; and quality of life. ${ }^{17}$

In DSM-5, a depressive episode with MFS would apply if the criteria for an MDE are met along with 3 or more of the following nonoverlapping symptoms: elevated/expansive mood, inflated self-esteem, talkativeness, flight of ideas, increased energy, goaldirected activity, decreased need for sleep, and excessive involvement in activities that have high potential for adverse consequences. ${ }^{10}$

Major concerns still exist for the DSM-5 specifier particularly due to the exclusion of overlapping symptoms, such as distractibility, irritability, and psychomotor agitation. As a consequence, different classifications have been developed, such as the research-based diagnostic criteria for mixed depression (RBDC) that are defined by the presence of an MDE plus 3 out of 14 hypomanic symptoms. The included hypomanic symptoms differ between the 2 classifications: the DSM- 5 includes the symptom "decreased need for sleep" that is not included in the RBDC. The RBDC includes overlapping symptoms between episodes of opposite poles and other symptoms such as aggression and affective lability that are not considered in the rubric of mixed symptoms in the DSM-5.

In 2015, a large multicenter study, the Bipolar Disorders: Improving Diagnosis, Guidance and Education (BRIDGE)-II-Mix study, was conducted in order to estimate the frequency of depressive mixed features during an MDE, considering not only the DSM-5 criteria but also the RBDC criteria. The latter identified 4 times more patients with MDE as having mixed features and yielded statistically more robust associations with several illness characteristics of BD than did DSM-5 criteria, such as family history of mania; lifetime suicide attempts; duration of the current episode greater than 1 month; atypical features; early onset history of AD-induced mania/hypomania; and lifetime comorbidity with anxiety, alcohol, and substance use disorders, attention-deficit/hyperactivity disorder, and borderline personality disorder. ${ }^{18}$ 
A recent cluster analysis of the same study identified an algorithm of predictive symptoms of mixed depression and supported the predominant role for overlapping "manic" symptoms in defining mixed depressive states, namely irritability, psychomotor agitation, and distractibility, together with other excitatory features shared with mania and atypical features, such as emotional lability, mood reactivity, absence of reduced appetite, and absence of psychomotor retardation, rather than by nonoverlapping manic symptoms. ${ }^{19}$

\section{WHY DSM-5 DOES NOT FIT WITH REAL MIXED DEPRESSION?}

Several aspects of the clinical presentation of MDE with MFS commonly seen in clinical practice unfortunately seem not to be reflected by the diagnostic DSM- 5 criteria for mixed features. Particularly, it is quite infrequent to observe euphoria and grandiosity as mixed features in the context of an MDE both in bipolar and unipolar depression. Indeed, findings from the BRIDGE-II-Mix study highlighted that euphoria and grandiosity were rare mixed features (4.6\% and 3.7\%, respectively) among the entire sample. ${ }^{18}$ Similarly, Sato and colleagues ${ }^{20}$ found that in their sample euphoria and grandiosity ranged from $1 \%$ to $4 \%$ and stated that euphoria and grandiosity were too uncommon to be used for the selection of patients with depressive mixed states. Maj and colleagues ${ }^{21}$ reported that no depressive patients, either agitated or nonagitated, presented elevated mood, inflated self-esteem, or grandiosity. Results from the Systematic Treatment Enhancement Program for Bipolar Disorder, including data of 1380 bipolar depressed patients, found that in those patients presenting subsyndromal mania or a full mixed episode the specific manic symptoms with the highest frequency did not include either elation or grandiosity. ${ }^{22}$ These findings suggest that the "real-world" depressive patients with mixed features may have characteristics of hypo/manic behavior but they lack expansiveness typical of euphoria and grandiosity. $^{12}$

With the aim of avoiding overdiagnosis, DSM-5 MFS has excluded the overlapping "manic" symptoms, leaving many patients with mixed depression undiagnosed. ${ }^{12}$ Many investigators have claimed how overlapping symptoms, such as anxiety, psychomotor agitation, mood lability, irritability, and distractibility, are the most frequently observed in mixed patients and may actually represent the core features of mixed depression. ${ }^{12,23,24}$ In the BRIDGE-II-Mix study including 2811 unipolar or bipolar depressed patients, the most frequent manic/hypomanic symptoms according to the RBDC mixed criteria were irritable mood (32.6\%), emotional/mood lability (29.8\%), distractibility (24.4\%), psychomotor agitation (16.1\%), impulsivity $(14.5 \%)$, aggression $(14.2 \%)$, racing thoughts $(11.8 \%)$, and pressure to keep talking $(11.4 \%) .{ }^{18}$ Interestingly, in a recent cluster analysis of the BRIDGE-II-Mix study, although nonoverlapping DSM-5 mixed symptoms were more represented among the group of depressive patients with mixed features, the symptoms that better predicted the mixed cluster were irritable mood, emotional/mood lability, psychomotor agitation, distractibility mood reactivity, absence of reduced appetite, and absence of psychomotor retardation, pointing out that none of these correspond to DSM-5 MFS criteria. ${ }^{19}$

Noteworthy, most of the overlapping symptoms have been found to be associated with clinical and course variables related to a bipolar diathesis such as BD diagnosis, family history of hypomania, atypical features, early age of onset, history of switch with ADs, suicide attempts, comorbidity with alcohol and substances disorders, attention deficit hyperactivity disorder (ADHD) and borderline personality disorder, probably indicating a better "affinity" of these features to the bipolar spectrum. ${ }^{18}$ Similarly, Malhi 
and colleagues ${ }^{23}$ found that the so-called DIP symptoms, including distractibility, irritability, and psychomotor agitation, were fundamental to define mixed depression and all 3 were key features in indicating a clinical course that suggests a bipolar spectrum disorder.

This leads to another important issue: the capability of the DSM-5 to capture the real accurate diagnosis of mixed presentations. In fact, overlapping criteria have been excluded by DSM-5 because they were thought to be nonspecific. ${ }^{23}$ As a consequence, the choice of a more "specific" approach has been made at the expenses of the "sensitivity" of the classification. ${ }^{25,26}$ In fact, DSM-5 MFS presented a $100 \%$ specificity but only $5.1 \%$ sensitivity, ${ }^{27}$ as also highlighted in the recent cluster analysis of the BRIDGE-II-Mix group. ${ }^{19}$ This means that up to $95 \%$ of patients presenting with mixed features according to the DSM- 5 are wrongly diagnosed as having "pure" affective episodes with huge clinical and therapeutic implications. ${ }^{25,27}$

The speculative wish to avoid "overlapping" symptoms, such as psychomotor agitation, irritability, and mood lability, the most common features of mixed depression across the literature, is very restrictive, allowing the diagnosis of mixed depression only in 1 out of 4 cases. ${ }^{18}$ Certainly, these symptoms may be nonspecific but to exclude them entirely may be not justified, in the absence of any evidence that the remaining criteria are sufficiently sensitive. ${ }^{28}$

\section{THE CLINICAL RELEVANCE OF MIXED SYMPTOMS NOT INCLUDED IN DSM-5 CRITERIA: RESULTS FROM BRIDGE-II-MIX STUDY Psychomotor Agitation}

DSM-5 criteria for MFS currently exclude psychomotor agitation, making it diagnostically irrelevant because it represents one of the symptoms defining the $B$ criterion of an MDE in DSM-5. Conversely, the concept of agitation and psychomotor excitement has been consistently proposed as the key point of mixed depression across the literature. Actually, Koukopoulos and colleagues, ${ }^{12,16}$ based on their clinical experience, considered the classic "agitated depression" the most dramatic clinical picture of depressive mixed states, being psychomotor agitation, when present, a fairly distinctive symptom to make the diagnosis of mixed depression. Moreover, in a pooled post hoc analysis of both the BRIDGE and the BRIDGE-II-Mix studies, psychomotor agitation was closely related to bipolarity and was found to characterize an especially difficult-to-treat subgroup of patients, requiring a more complex regimen of pharmacologic treatment. ${ }^{29}$

\section{Affective Lability}

The psychopathologic construct of affective lability assumed a central role both as a traitlike clinical feature in mixed episodes ${ }^{30}$ and as one of the 3 most frequent state features in mixed depression, together with agitation and irritability. ${ }^{12}$ Traditionally, affective lability in a depressive mixed episode was considered as a risk factor of shifting between MDD and $\mathrm{BD}^{31,32}$ and was found to be strongly associated with atypical depression, in which the core symptom is mood reactivity, ${ }^{10}$ that has been typically related to bipolar depression. ${ }^{32}$

In a post hoc analysis of the BRIDGE-II-Mix study, ${ }^{33}$ more than a half of the patients reporting affective lability were diagnosed with a DSM-5 MDE with mixed features. Several findings from this study seem to support that the presence of affective lability during an MDE was associated with mixicity, particularly with a more severe clinical condition, such as the severity of cooccurring hypo/manic symptoms during an MDE. Furthermore, mood reactivity was the variable most significantly associated 
with affective lability, and vice versa, leading the investigators to review their results in the light of a unique continuum between mood states, concluding that the intertwined association between affective lability and mood reactivity might bridge the gap between mixed and atypical depression. ${ }^{33}$ Consistently, affective lability and mood reactivity are 2 of the 7 clinical features proved to be the best predictors of membership of the mixed cluster in the cluster analysis of the BRIDGE-II-Mix study. ${ }^{19}$

\section{Aggressiveness}

Aggressiveness is not currently considered as a DSM diagnostic criterion of MFS in either bipolar or unipolar depressive episodes despite its prevalence being around $15 \%$ in an MDE, according to RBDC mixed criteria. ${ }^{18}$ In a post hoc analysis of the BRIDGE-II-Mix study ${ }^{34}$ focused on this behavioral construct, the most relevant clinical variable associated with aggressiveness was the presence of a DSM-5 MDE with mixed features. Several findings from this study supported its association with bipolarity per se, independently from comorbid disorders such as borderline personality disorder and substance abuse, for example, the higher frequency of BD diagnosis in depressed patients with aggressiveness, whereas a diagnosis of unipolar depression was negatively correlated with aggressive behaviors. Moreover, MDE patients with aggressiveness showed higher rates of family history for BD as well as younger age at the first depressive episode. Taken together, these results might have important implications in terms of the reconsideration of aggressiveness for diagnostic criteria for the MFS.

The proposed mixed features are shown in Fig. 1.

\section{CLINICAL INDICATORS OF “MIXICITY" THAT SUGGEST A BIPOLAR DIATHESIS}

Interestingly, the RBDC criteria, but not DSM-5 criteria, for MFS were found to be associated with several indicators of bipolarity, as mentioned earlier. ${ }^{18}$

Since the publication of the BRIDGE-II-Mix study, several post hoc analyses found that different clinical variables related to a bipolar diathesis were more likely associated with the presence of mixed features during a bipolar or unipolar MDE (Fig. 2).

\section{Suicidality}

The presence of suicidality was the focus of a post hoc analysis by Popovic and colleagues, ${ }^{35}$ which found that depressed patients with a previous history of suicide attempts presented distinct clinical features that suggest a bipolar diathesis, such as a first-degree family history of BD, psychotic and atypical features, more (hypo)manic switches with $A D$, and higher rates of treatment resistance. Moreover, suicidal

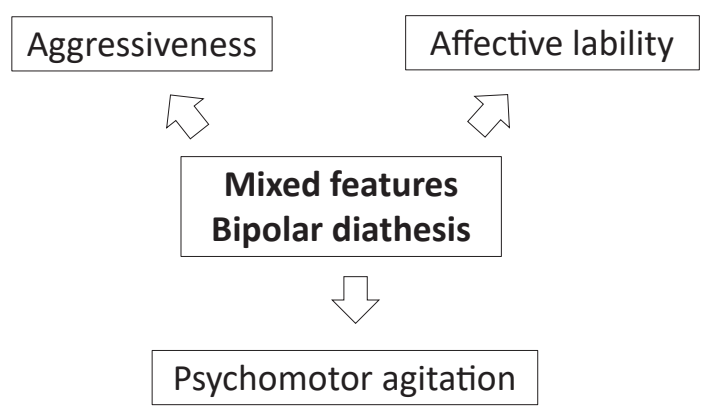

Fig. 1. New proposed mixed features: results from BRIDGE-II-Mix study. 


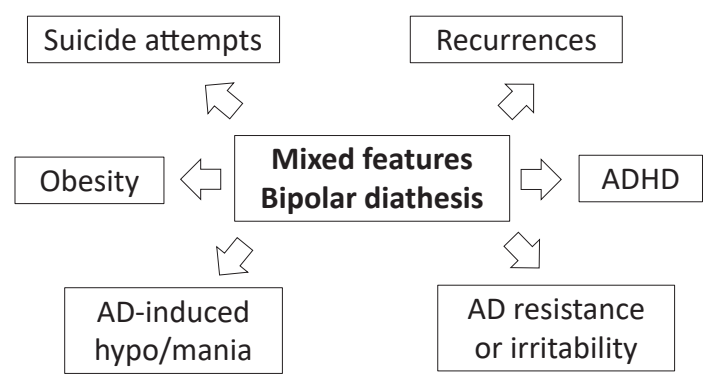

Fig. 2. Clinical and course features that suggest BD: results from BRIDGE-II-Mix study.

behavior was associated with RBDC mixed features, particularly risky behavior, psychomotor agitation, and impulsivity.

\section{Antidepressant-Induced Hypomania}

The emergence of new hypo/manialike responses to AD treatment in patients diagnosed as having a major depressive disorder (MDD-AIHM) has been assessed by Barbuti and colleagues. ${ }^{36}$ The investigators compared patients in the MDD-AlHM group with those suffering from MDD or BD without a history of AD-induced hypo/mania and found that in the MDD-AIHM group, familiarity for BD and rates of atypical features and comorbid psychiatric disorders were similar to that of patients with BD and significantly more frequent compared with the MDD group. MDD-AIHM patients presented clinical course variables associated with BD, such as more than 3 affective episodes and higher rates of treatment resistance. The frequency of MDE with MFS was similar in patients of the MDD-AIHM and BD groups and significantly higher in both groups than in the MDD group. This led the investigators to claim the need for the DSM-5 inclusion of patients with MDD with AIHM within the rubric of BD.

\section{Treatment Resistance or Worsening of Depression Associated with Antidepressant}

Another recent analysis from the BRIDGE-II-Mix group was aimed at exploring the possible association between different inadequate patterns of response to ADs and bipolarity in unipolar depressive patients. ${ }^{37}$

Patients with or without a history of resistance to $A D$ treatment and with or without a previous $A D$-induced irritability and mood lability have been compared. Those with $A D$ treatment resistance showed higher rates of psychotic features, history of suicide attempts, emotional lability and impulsivity as mixed features, comorbid borderline personality disorder, and polypharmacologic treatment.

Patients presenting previous affective lability and irritability following $A D$ treatment showed significantly higher rates of first-degree family history for BD, previous treatment-resistant depression, atypical features, psychiatric comorbidities, lifetime suicide attempts, and lower age at first psychiatric symptoms. Among mixed features, distractibility, impulsivity, and hypersexuality were significantly associated with worsening of depression with AD. The investigators concluded that in unipolar depressed patients, a lifetime history of resistance and/or irritability/mood lability in response to ADs was associated with the presence of mixed features and a possible underlying bipolar diathesis.

\section{Higher Rates of Depressive Recurrences}

The presence of mixed features frequency was assessed comparing unipolar depressive patients with high versus low recurrences rates. ${ }^{38}$ Patients with higher 
recurrences presented more hypomanic symptoms during current MDE, resulting in higher rates of mixed depression according to both DSM-5 and RBDC criteria. Moreover, they presented clinical indicators of bipolarity such as earlier depressive onset, more family history of BD, more atypical features, suicide attempts, treatment resistance, (hypo)manic switches when treated with $A D$, and higher psychiatric comorbidities.

\section{Obesity}

A comparison of patients with or without obesity (body mass index $>30$ ) presenting an MDE was conducted in a subanalysis of the BRIDGE-II-Mix study. ${ }^{39}$ Obese depressed patients showed higher rates of history of (hypo)manic switches during AD treatment, atypical depressive features, comorbid eating disorders, and anxiety disorders. Psychomotor agitation, distractibility, increased energy, and risky behaviors were the mixed features more frequently presented among obese patients. When hypersomnia was studied with the metabolic aspects in the same sample, the cooccurrence of hypersomnia and overweight/obesity represented a more extreme clinical phenotype of bipolarity complicated by treatment-related adverse effects and mixed features. ${ }^{40}$

\section{Psychiatric Comorbidities}

The presence of psychiatric comorbidities, particularly those sharing an important underpinning impulsive component, has been related in a sample of depressed patients with mixed features and bipolarity. The presence of mixed features during an MDE in comorbidity with borderline personality disorder was associated with a more complex course of illness associated with bipolarity, reduced treatment response, and worse outcomes. ${ }^{41}$ Similarly, in those patients presenting a comorbid ADHD, mixed features and bipolar clinical course indicators were more represented. ${ }^{42}$

\section{SUMMARY}

A proper identification of an MDE with mixed features is of huge importance not only for the diagnostic implications but also for clinical practice due to the worse course and treatment issues associated with this condition.

The DSM-5 made an important step in this sense by introducing the specifier "with mixed features," thus recognizing for the first time that symptoms of opposite polarity may co-occur during an MDE of either BD-I, BD-II, or unipolar MDD and are diagnostically different from pure episodes within these 3 clinical entities. Conversely, the DSM-5 criteria for MFS during an MDE are based on the putative construct of avoiding overdiagnosis due to overlapping symptoms. The definition of MDE with MFS according to DSM-5 is controversial because it includes typical manic symptoms, such as elevated mood and grandiosity, that were found to be rare among patients with mixed depression, whereas it excludes overlapping excitatory symptoms that are frequently reported in mixed depression. Leaving out these 3 symptoms means that only $25 \%$ of patients with mixed depression will actually meet DSM- 5 criteria. In this sense, the authors agree with Koukopoulos and Sani's position that is to rename the clinical identity of these symptoms as "excitatory" instead of "manic," in order to underline the different nature of this excitatory component from the pure manic symptom presentation in defining mixed features during an MDE.

Indeed, the BRIDGE-II-Mix group claimed at including in the rubric of MFS symptoms, such as psychomotor agitation, mood lability, and aggressiveness, which are not currently considered as mixed features in the DSM-5. 
Furthermore, despite the DSM-5 MFS may characterize also a unipolar mixed depression, we argue that the presence of excitatory overlapping symptoms may describe more specifically a subgroup of depressed patients uncovering a bipolar diathesis. This is in line with the findings of the studies mentioned earlier, in which several clinical and course indicators of bipolarity, such as suicidality, AD-induced hypo(mania), treatment resistance or worsening of depression associated with $A D$, higher rates of depressive recurrences, metabolic issues such as overweight or obesity, and the presence of specific psychiatric comorbidities, ADHD, and borderline personality disorder in particular were found to be associated with the presence of mixed characteristics, mainly overlapping excitatory symptoms, during an MDE.

The aforementioned considerations suggest the need of a deep revision of the MFS in depression for the planned DSM-5 text revision.

\section{DISCLOSURE}

Dr I. Pacchiarotti has received CME-related honoraria or consulting fees from ADAMED, Janssen-Cilag, and Lundbeck. Dr A. Murru has received grants, honoraria, or consulting fees from, Janssen, Lundbeck, and Otsuka. Prof. G. Perugi has acted as consultant of Lundbeck, Angelini, FB-Health. He received grant/research support from Lundbeck and Angelini. He is on the speaker/advisory board of Sanofi-Aventis, Lundbeck, FB-Health, and Angelini. Pr E. Vieta has received grants and served as consultant, advisor, or CME speaker for the following entities: AB-Biotics, Abbott, Allergan, Angelini, AstraZeneca, Bristol-Myers Squibb, Dainippon Sumitomo Pharma, Farmindustria, Ferrer, Forest Research Institute, Gedeon Richter, GlaxoSmithKline, Janssen, Lundbeck, Otsuka, Pfizer, Roche, SAGE, sanofi-aventis, Servier, Shire, Sunovion, Takeda, the Brain and Behaviour Foundation, the Spanish Ministry of Science and Innovation (CIBERSAM), the EU Horizon 2020, and the Stanley Medical Research Institute. Dr G.D. Kotzalidis, Dr L. Mazzarini, Dr C. Rapinesi, Dr M. Valentí, Dr G. Anmella, Dr S. Gomes-da-Costa, Dr A. Gimenez, Dr C. Llach, and Dr N. Verdolini has been supported by a BITRECS. BITRECS project has received funding from the European Union's Horizon 2020 research and innovation programme under the Marie Skłodowska-Curie grant agreement No 754550 and from "La Caixa" Foundation.

\section{REFERENCES}

1. Grande I, Berk M, Birmaher B, et al. Bipolar disorder. Lancet 2016;387(10027): 1561-72.

2. Solé E, Garriga M, Valentí M, et al. Mixed features in bipolar disorder. CNS Spectr 2017;1-7. https://doi.org/10.1017/S1092852916000869.

3. Vieta E, Berk M, Schulze TG, et al. Bipolar disorders. Nat Rev Dis Prim 2018;4: 18008.

4. Vieta E, Salagre E, Grande I, et al. Early intervention in bipolar disorder. Am J Psychiatry 2018;175(5):411-26.

5. American Psychiatric Association. Diagnostic and statistical manual of mental disorders 3rd edition. Washington, DC; 1980.

6. American Psychiatric Association. Diagnostic and statistical manual of mental disorders. 3rd edition, Rev. Washington, DC: Author.; 1987.

7. American Psychiatric Association. Diagnostic and statistical Manual of mental disorders. 4th edition. Washington, DC: Author.; 1994.

8. American Psychiatric Association. Diagnostic and statistical manual of mental disorders. 4th edition, text revision. Washington, DC: Author.; 2000. 
9. World Health Organization. International statistical classification of diseases and related health problems, 10th Revision (ICD-10). Geneva (Switzerland): Author.; 1992.

10. American Psychiatric Association. Diagnostic and statistical manual of mental disorders. 5th edition. Washington, DC: American Psychiatric Association; 2013.

11. Takeshima M, Oka T. DSM-5-defined 'mixed features' and Benazzi's mixed depression: Which is practically useful to discriminate bipolar disorder from unipolar depression in patients with depression? Psychiatry Clin Neurosci 2015; 69(2):109-16.

12. Koukopoulos A, Sani G. DSM-5 criteria for depression with mixed features: a farewell to mixed depression. Acta Psychiatr Scand 2014;129(1):4-16.

13. Angst J, Cui L, Swendsen J, et al. Major depressive disorder with subthreshold bipolarity in the national comorbidity survey replication. Am J Psychiatry 2010; 167(10):1194-201.

14. Smith DJ, Forty L, Russell E, et al. Sub-threshold manic symptoms in recurrent major depressive disorder are a marker for poor outcome. Acta Psychiatr Scand 2009;119(4):325-9.

15. Zimmermann P, Brückl T, Nocon A, et al. Heterogeneity of DSM-IV major depressive disorder as a consequence of subthreshold bipolarity. Arch Gen Psychiatry 2009;66(12):1341.

16. Koukopoulos A, Sani G, Koukopoulos AE, et al. Melancholia agitata and mixed depression. Acta Psychiatr Scand Suppl 2007;115(433):50-7.

17. Mclntyre RS, Soczynska JK, Cha D, et al. The prevalence and illness characteristics of DSM-5-defined "mixed feature specifier" in adults with major depressive disorder and bipolar disorder: results from the International Mood Disorders Collaborative Project. J Affect Disord 2015;172:259-64.

18. Perugi G, Angst J, Azorin J-M, et al. Mixed features in patients with a major depressive episode: the BRIDGE-II-MIX study. J Clin Psychiatry 2015;76(3): e351-8.

19. Brancati GE, Vieta E, Azorin J-M, et al. The role of overlapping excitatory symptoms in major depression: are they relevant for the diagnosis of mixed state? J Psychiatr Res 2019;115:151-7.

20. Sato T, Bottlender R, Schröter A, et al. Frequency of manic symptoms during a depressive episode and unipolar "depressive mixed state" as bipolar spectrum. Acta Psychiatr Scand 2003;107(4):268-74. Available at: http://www.ncbi.nlm.nih. gov/pubmed/12662249. Accessed July 11, 2018.

21. Maj M, Pirozzi R, Magliano L, et al. Agitated "unipolar" major depression: prevalence, phenomenology, and outcome. J Clin Psychiatry 2006;67(5):712-9. Available at: http://www.ncbi.nlm.nih.gov/pubmed/16841620. Accessed July 21, 2019.

22. Goldberg JF, Perlis RH, Bowden CL, et al. Manic symptoms during depressive episodes in 1,380 patients with bipolar disorder: findings from the STEP-BD. Am J Psychiatry 2009;166(2):173-81.

23. Malhi GS, Byrow Y, Outhred T, et al. Exclusion of overlapping symptoms in DSM-5 mixed features specifier: heuristic diagnostic and treatment implications. CNS Spectr 2017;22(2):126-33.

24. Perugi G, Quaranta G, Dell'Osso L. The significance of mixed states in depression and mania. Curr Psychiatry Rep 2014;16(10):486.

25. Mclntyre RS, Lee Y, Mansur RB. A pragmatic approach to the diagnosis and treatment of mixed features in adults with mood disorders. CNS Spectr 2016; 21(S1):25-33. 
26. Vieta E, Valentí M. Mixed states in DSM-5: Implications for clinical care, education, and research. J Affect Disord 2013;148(1):28-36.

27. Stahl SM. Mixed-up about how to diagnose and treat mixed features in major depressive episodes. CNS Spectr 2017;22(02):111-5.

28. Perugi G. ICD-11 mixed episode: nothing new despite the evidence. Bipolar Disord 2019;21(4):376-7.

29. Barbuti M, Mainardi C, Pacchiarotti I, et al. The role of different patterns of psychomotor symptoms in major depressive episode: pooled analysis of the BRIDGE and BRIDGE-II-MIX cohorts. Bipolar Disord 2019. [Epub ahead of print].

30. Mackinnon DF, Pies R. Affective instability as rapid cycling: theoretical and clinical implications for borderline personality and bipolar spectrum disorders. Bipolar Disord 2006;8(1):1-14.

31. Akiskal HS, Maser JD, Zeller PJ, et al. Switching from "unipolar" to bipolar II. An 11-year prospective study of clinical and temperamental predictors in 559 patients. Arch Gen Psychiatry 1995;52(2):114-23.

32. Benazzi F. The relationship of major depressive disorder to bipolar disorder: continuous or discontinuous? Curr Psychiatry Rep 2005;7(6):462-70. Available at: http://www.ncbi.nlm.nih.gov/pubmed/16318825. Accessed July 21, 2019.

33. Verdolini N, Menculini G, Perugi G, et al. Sultans of swing: a reappraisal of the intertwined association between affective lability and mood reactivity in a post hoc analysis of the BRIDGE-II-MIX study. J Clin Psychiatry 2019;80(2). https:// doi.org/10.4088/JCP.17m12082.

34. Verdolini N, Perugi G, Samalin L, et al. Aggressiveness in depression: a neglected symptom possibly associated with bipolarity and mixed features. Acta Psychiatr Scand 2017. https://doi.org/10.1111/acps.12777.

35. Popovic D, Vieta E, Azorin J-M, et al. Suicide attempts in major depressive episode: evidence from the BRIDGE-II-Mix study. Bipolar Disord 2015;17(7): 795-803.

36. Barbuti M, Pacchiarotti I, Vieta E, et al. Antidepressant-induced hypomania/ mania in patients with major depression: evidence from the BRIDGE-II-MIX study. J Affect Disord 2017;219:187-92.

37. Perugi G, Pacchiarotti I, Mainardi C, et al. Patterns of response to antidepressants in major depressive disorder: drug resistance or worsening of depression are associated with a bipolar diathesis. Eur Neuropsychopharmacol 2019; 29(7):825-34.

38. Mazzarini L, Kotzalidis GD, Piacentino D, et al. Is recurrence in major depressive disorder related to bipolarity and mixed features? Results from the BRIDGE-II-Mix study. J Affect Disord 2018;229:164-70.

39. Petri $E$, Bacci $O$, Barbuti $M$, et al. Obesity in patients with major depression is related to bipolarity and mixed features: evidence from the BRIDGE-II-Mix study. Bipolar Disord 2017;19(6):458-64.

40. Murru A, Guiso G, Barbuti M, et al. The implications of hypersomnia in the context of major depression: results from a large, international, observational study. Eur Neuropsychopharmacol 2019;29(4):471-81.

41. Perugi G, Angst J, Azorin J-M, et al. Relationships between mixed features and borderline personality disorder in 2811 patients with major depressive episode. Acta Psychiatr Scand 2016;133(2):133-43.

42. Vannucchi G, Medda P, Pallucchini A, et al. The relationship between attention deficit hyperactivity disorder, bipolarity and mixed features in major depressive patients: Evidence from the BRIDGE-II-Mix Study. J Affect Disord 2019;246: 346-54. 